\title{
Zur aktuellen Situation im Umgang mit der Corona- Pandemie in einer Universitatsklinik
}

Wolfgang Toller, befragt von Wolfgang Kröll

Kröll: Zuerst, lieber Wolfgang, vielen Dank, dass Du Dich trotz Deiner zahlreichen und vielfältigen Aufgabenbereiche bereit erklärt hast, mir einige Fragen retro- und prospektiv zur Corona-Pandemie zu beantworten. Darf ich Dich zuerst etwas sehr Persönliches fragen? Wie geht es Dir persönlich? Bist Du oder sind Mitglieder Deiner Familie an COVID-19 erkrankt? Bist Du oder jemand aus Deiner Familie bereits geimpft? Darf ich Dich auch fragen, welcher Impfstoff Dir verabreicht wurde? Und, wie waren Deine persönlichen Erfahrungen mit der Impfung?

Toller: Persönlich geht es mir sehr gut; ich hatte vor Kurzem eine Operation, habe diese aber auch gut überstanden und fühle mich wohl. Bezüglich COVID-19 war ich als angestellter Arzt des LKH-Universitätsklinikums Graz in der glücklichen Lage bereits am 8.1. und am 29.1.2021 meine beiden Impfdosen von Pfizer erhalten zu haben; dennoch ist es bei uns am LKH-Universitätsklinikum in Graz weiterhin Usus, dass die Mitarbeiter zumindest einmal wöchentlich einen Antigen-Test machen müssen und auch bei diesen Tests wurde ich immer negativ getestet.

Innerhalb meiner Familie ist meine Frau an COVID-19 erkrankt; sie hat sich bei einem Nachbarn infiziert; glücklicherweise ist die Erkrankung milde verlaufen. Für mich war in diesem Zusammenhang positiv, dass ich erkennen konnte, dass meine Impfung tatsächlich sehr gut wirkt. Da ich in der Zeit eine „Verkehrsbeschränkung“, aber keine Quarantäne hatte, führten wir bei mir im Spital häufig einen SARS-CoV2-Test durch, ich wurde aber niemals positiv getestet. Und dies, obwohl ich bzw. wir beide zuhause sicherlich keinen allzu großen Abstand eingehalten hatten. Umso mehr bin ich darüber erfreut, dass ich diese Impfung schon sehr bald nach Freigabe des Impfstoffs durch die EMA erhalten habe.

Innerhalb meiner Familie ist es so, dass meine Frau zum Zeitpunkt des Ausbruchs der SARS-CoV2-Infektion leider noch nicht geimpft war und, soweit jetzt bekannt, bis zu einem möglichen Impftermin noch fünf Monate warten muss. Unser jüngerer Sohn (26 Jahre) ist noch nicht geimpft, unser älterer Sohn (28 Jahre) ist in der Pharmaindustrie tätig und hat von 
seinem Dienstgeber aus auch bereits die Impfung erhalten. Die Impfung selbst war weder für mich noch für meinen Sohn in irgendeiner Weise belastend; nur an der Stelle des Oberarms, an der die beiden Impfungen appliziert worden sind, verspürte ich jeweils für einen Tag einen geringen Druckschmerz; andere Symptome waren bei keiner der beiden Impfdosen zu bemerken.

Kröll: Die Corona-Pandemie dauert nun auch in Österreich schon länger als ein Jahr und sie hat die Welt und die Gesellschaft in vielerlei Weise verändert. Wie hast Du das persönlich wahrgenommen? Was hat sich für Dich in Deiner Familie, in Deinem privaten und persönlichen Umfeld verändert? Aus Deiner persönlichen Sicht: Wie viele dieser Veränderungen werden auch nach Beendigung der Pandemie noch Bestand haben? Wie werden sich aus Deiner Sicht die Welt und die Gesellschaft nach dem Ende der Pandemie präsentieren? Wird kein Stein mebr auf dem anderen bleiben?

Toller: Persönlich habe ich die Corona-Pandemie sehr ambivalent empfunden: Auf der einen Seite war es interessant zu beobachten, wie leicht man auf viele Dinge, von denen man überzeugt war, dass man sie im Alltag unbedingt benötigt, verzichten und sich auf wirkliche Werte konzentrieren kann; auf der anderen Seite waren da natürlich die vorgegebenen Einschränkungen und in diesem Kontext hat man natürlich schon auch erkannt, dass es für jeden einzelnen Menschen Bedürfnisse gibt, die von großer Bedeutung sind, obwohl man diese im „Normalfall“ nicht großartig erwähnen würde: z. B. der Kontakt zur Familie. Die Tatsache, dass man Eltern oder nahe Angehörige nicht besuchen konnte, unsere Kinder selten zu Besuch kamen und Kontakt zumeist nur digital oder per Telefon stattfand, war belastender, als ich es mir vorgestellt habe. Solche Gegebenheiten möchte ich in dieser Form nicht noch einmal erleben.

Ich bin überzeugt, dass einige der Veränderungen, die durch die Corona-Pandemie angestoßen wurden, nach ihrer erfolgreichen Bekämpfung bestehen bleiben werden. Ich bin auch überzeugt, dass die Corona-Pandemie nicht so schnell beendet sein wird. Man wird bei Reisen ins Ausland alert sein müssen, welche Voraussetzungen zur Einreise und zum Aufenthalt einzuhalten sind. Denn das SARS-CoV2 wird nicht von der Bildfläche verschwinden, so wie wir uns dies gerne wünschen und vorstellen würden; vielmehr wird dieses Virus weiter existieren, auch in Mutationen, ähnlich dem Grippevirus. Und die Impfung, die Auffrischungsimpfung, die Antigen-Tests oder auch die PCR-Tests werden auch weiterhin erforderlich sein, um eine gelungene Eindämmung der Ausbreitung beibehalten zu können. Denn ich bin auch überzeugt, kein Staat wird es sich in 
Zukunft leisten können, noch einmal die gesamten Wirtschaftsbereiche, den Tourismus etc. über so lange Zeiträume niederzufahren bzw. weitere Lockdowns anzuordnen.

Kröll: Du leitest die zweitgrößte Universitätsklinik für Anästhesiologie und Intensivmedizin, wahrscheinlich auch eine der größten Universitätskliniken für diesen Fachbereich im deutschsprachigen Raum: Wie hast Du die Corona-Pandemie in Deinem Bereich aus ärztlicher Sicht gesehen? Ist die Krise über euch als Krankenanstalt hereingebrochen oder war das LKH-Universitätsklinikum darauf vorbereitet? Hat es Katastrophenpläne zur Bewältigung dieser Situation gegeben? Hattest Du in Deinem Bereich ausreichend Schutzausrüstungen sowobl für das ärztliche als auch für das nicht-ärztliche Gesundheitspersonal?

Toller: Als Leiter der Univ.-Klinik für Anästhesiologie und Intensivmedizin habe ich die Pandemie als sehr große Herausforderung empfunden. Das hängt einerseits damit zusammen, dass wir in der Steiermark das Zentralkrankenhaus bzw. das Universitätsklinikum sind und somit die Patienten mit den schwersten COVID-19-Verläufen bekamen, andererseits es sich aber auch sehr bald nach Ausbruch der Pandemie herausstellte, dass das grundsätzlich für COVID-19 zuständige Fachgebiet, nämlich die Univ.-Klinik für Innere Medizin, die intensivmedizinischen Ressourcen zur Betreuung aller dieser Patienten nicht haben würde und auch die chirurgischen Intensivstationen in die Behandlung und die Betreuung von COVID-19-Patienten eingebunden werden müssen.

Konkret bedeutete das, dass in der ersten Phase der Pandemie im März 2020 eine der beiden chirurgischen Herz-Intensivstationen ausschließlich für COVID-19-Patienten freigemacht werden musste und über viele Monate hinweg ausschließlich für diese Patienten zur Verfügung stand. Und in der Phase II, also Ende 2020 und zu Beginn 2021 wurde eine andere chirurgische Intensivstation, nämlich die ICU3, für COVID-19-Patienten adaptiert.

Obwohl in den Medien immer wieder berichtet wird, dass Österreich im Vergleich zu Italien, wo das SARS-CoV2 das erste Mal in Europa aufgetreten ist, Vorlaufzeiten für eine Adaptation hatte, ist die Pandemie im wahrsten Sinn des Wortes auch über unser Land hereingebrochen, nämlich in der Form, dass man gemerkt hat, dass theoretische Katastrophenszenarien, wie man sie in der Vergangenheit durchgespielt hatte, mit der Realität vielfach nicht übereinstimmten und man in kürzester Zeit auf einem völlig anderen Niveau Kooperationen in unterschiedlichen Bereichen suchen und eingehen musste und man damit völliges Neuland betreten hatte. 
So wurde zum Beispiel eine Normalstation geschlossen, die nicht in der Versorgung von COVID-19-Patienten involviert war, damit aus diesem Bereich Pflegepersonal auf jenen Stationen eingesetzt werden konnte, auf denen die zum Teil sehr pflegeaufwändigen COVID-19-Patienten zu behandeln und zu betreuen waren. Aus meiner Sicht wäre ein solches Vorgehen vor der Pandemie undenkbar gewesen, und war auch nie Bestandteil von immer wieder durchgeführten Katastrophenübungen. Auch arbeitszeitrechtlich war es für mich nicht vorstellbar, dass man in kürzester Zeit vollkommen neue Dienstmodelle installieren konnte.

Auch innerhalb der eigenen Klinik und Abteilung habe ich erlebt, welche täglichen Probleme im Rahmen einer Pandemie auf uns zukommen können. Als Leiter einer Klinik bzw. Abteilung ist man für seine Mitarbeiter verantwortlich und macht sich selbstverständlich einerseits Sorgen um ihre Gesundheit, muss aber andererseits auch die Behandlung von COVID-19- und anderen Patienten gewährleisten. Unter den ärztlichen Mitarbeitern haben sich manche freiwillig gemeldet, um auf einer COVID-19-Station arbeiten zu dürfen und dort auch engagiert ihre Leistung erbracht, andere kamen mit Attesten, die ihnen bescheinigt haben, dass sie aus diversen Gründen nicht in der Lage sind auf einer solchen Station ihren Dienst zu versehen, andere erkrankten selbst an COVID-19 oder wurden als Kontaktpersonen von den Behörden in Quarantäne geschickt. Einen 24/7-Dienstplan unter solchen Bedingungen zu erstellen ist nicht einfach, man musste sich täglich auf neue Situationen einstellen und flexibel darauf reagieren. Über die sozialen Medien, wie WhatsApp etc., in denen die Mitarbeiter auch häufig untereinander kommunizierten, war es aber meist möglich, bei Ausfall eines Kollegen relativ bald wieder einen anderen Kollegen zu finden, der bereit war einen Nachtdienst zu übernehmen.

Die erforderliche Ausrüstung war, wie auch in anderen europäischen Staaten, am Anfang der Pandemie nicht ausreichend: Das betrifft im Besonderen die Schutzausrüstungen, die Schutzmasken bis hin zu den Schutzanzügen. Es gab tatsächlich die in den Medien kolportierten Lieferverzögerungen und Lieferengpässe, speziell bei FFP2-Masken. Das hat dann dazu geführt, dass insbesondere beim Pflegepersonal die Masken mehr oder weniger während einer gesamten Dienstschicht getragen werden mussten. Im Herbst und Winter 2020 war dann ausreichend Schutzmaterial für das gesamte Personal verfügbar und es war auch kein Engpass mehr zu bemerken. 
Kröll: Wie hat sich die Corona-Pandemie auf Deine ärztlichen Mitarbeiter ausgewirkt? Welche belastenden Ereignisse wurden bei der Behandlung dieser Patienten von den Ärzten wahrgenommen? Welche Unterstützung haben die Mitarbeiter vom Arbeitgeber in dieser Situation erhalten? Welche psychosozialen Unterstützungsangebote würden sie sich noch wünschen? Sind Deine ärztlichen Mitarbeiter noch immer so motiviert bei der Behandlung von SARS-CoV2-infizierten Patienten wie zu Beginn der Krise oder sind entsprechende und verständliche Ermüdungserscheinungen und Demotivation zu beobachten?

Toller: Die Belastung für die Mitarbeiter war enorm; wie tatsächlich die mittel- und langfristigen Folgen dieser Belastung ausfallen werden, lässt sich derzeit noch gar nicht abschätzen. Aber wie auch in anderen klinischen Bereichen so zeichnet sich auch in der Anästhesiologie ab, dass einige Mitarbeiter eine berufliche Veränderung anstreben; dies wird an unserer Klinik vermutlich im Herbst 2021 zu einem Engpass an ärztlichen Mitarbeitern führen; zurückzuführen ist dies u. a. auch darauf, dass einige unserer Mitarbeiter, die aus dem nahem Ausland kommen, täglich stundenlang im Auto sitzen mussten, bei der Einreise in die Steiermark Verzögerungen erleben mussten etc. und dies in Zukunft einfach nicht mehr mitmachen wollen.

Die Versorgung mit Schutzausrüstungen hat sehr rasch und sehr gut funktioniert. Die Notwendigkeit Schutzanzüge anziehen zu müssen hat sich nicht unbedingt als Problem herausgestellt, zumindest nicht beim Pflegepersonal. Bei den Ärzten war es vor allem der Journaldienst, der mit dem ständigen Wechseln des Schutzanzuges nach jedem Patientenkontakt schon eine enorme Belastung dargestellte. Eine Kollegin musste während eines sehr aufwändigen Journaldienstes durch Einschleusen in Isolationskojen insgesamt 32-mal ihre Schutzausrüstung wechseln. Ich selbst habe dennoch den Eindruck, dass der Umgang mit SARS-CoV2-Patienten im heurigen Jahr etwas „lockerer“ geworden ist, als dies zu Beginn der Fall war, wo ja nicht bekannt war, was uns bei der Behandlung und Betreuung dieser Menschen erwarten würde. Es ist derzeit vieles einfacher durchzuführen, z. B. die Adaptation einer Station derart, dass in einem Teil der Station infizierte und in einem anderen Teil der Station postoperativ zu betreuende Patienten behandelt werden. Ein solches Vorgehen wäre zu Beginn der Pandemie undenkbar gewesen.

Bzgl. der psychischen Unterstützung der Mitarbeiter hat es Schreiben der Spitalsleitung gegeben, in welchen auf die Möglichkeit psychischer Unterstützung hingewiesen wurde. Insgesamt muss man zum Thema Information aber auch festhalten, dass speziell in der Anfangsphase der Pan- 
demie eine Flut an z. T. sich widersprechenden Informationen über die Mitarbeiter hereinbrach, die dann insgesamt eher zu Desinformation denn zu Information geführt hat. Irgendwann haben viele damit aufhört, alle ankommenden Informationsmails tatsächlich zu lesen. Vielleicht sollte man für eine zukünftige ähnliche Situation daraus lernen. Zusätzlich zu den internen Informationen und Regelungen kamen dann auch noch die Informationen aus öffentlichen Medien, die täglich über alle Staatsbürger ungefiltert hereingebrochen sind. Und wenn dann Informationen von einem auf den anderen Tag gültig bzw. wieder ungültig werden, dann darf man sich wohl kaum wundern, wenn bzgl. des Lesens dieser Informationen irgendwann Ermüdungserscheinungen auftreten. Und diese auch in den Medien kolportierten Ermüdungserscheinungen kann ich für die Univ.-Klinik für Anästhesiologie und Intensivmedizin nur bestätigen. Dennoch muss man auch als Vorgesetzter darauf achten, dass trotz der Informationsflut wenigstens jene Mitteilungen von den Mitarbeitern gelesen werden, die tatsächlich für den eigenen Schutz und für den Schutz der anvertrauten Patienten von Bedeutung sind.

Kröll: Soweit Du das beurteilen kannst: wie erging es dem Pflegepersonal auf jenen Intensivstationen, auf denen infizierte Patienten zu behandeln waren? Stand ausreichend Pflegepersonal für die Tätigkeit auf den Intensivstationen zur Verfügung oder war gerade die Pflege die knappe Ressource, die das Gesundheitssystem bei weiterer Ausbreitung der Corona-Pandemie hätte zum Kippen bringen können?

Toller: Auch beim Pflegepersonal war es ähnlich wie bei den ärztlichen Mitarbeitern; zu Beginn der Pandemie bestand ein sehr großer Respekt vor dem Virus und daraus resultierte auch eine große Unsicherheit sowohl bei den Ärzten als auch bei den Pflegepersonen. Das führte auch dazu, dass man eigenständige Intensivstationen ausschließlich zur Behandlung und Betreuung infizierter Patienten einrichtete. Im Laufe der Zeit hat sich dann auch eine gewisse Routine im Umgang mit dem Virus bzw. mit der Erkrankung entwickelt. Auch das Pflegepersonal stand nach einiger Zeit COVID-19-Patienten trotz der sehr hohen Belastung wesentlich entspannter gegenüber. Überrascht war ich auch über die Tatsache, dass das Pflegepersonal, das direkt in die Behandlung und Betreuung infizierter Patienten eingebunden war, dies weniger kritisch gesehen hatte als so manche Führungskräfte aus den unterschiedlichsten Bereichen. Die Belastung, besonders auch für das Pflegepersonal, war naturgemäß groß, dies war darauf zurückzuführen, dass COVID-19-Patienten ja nicht nur auf den Intensivstationen behandelt worden sind, sondern auch auf den 
Normalstationen. Und auf den Normalstationen war der Pflegeaufwand für COVID-19-Patienten beträchtlich größer als für die übrigen Patienten und daher war auch seitens des Pflegepersonals eine extrem hohe Flexibilität gefordert, z. B. dadurch, dass einzelne Stationen geschlossen worden sind und das Pflegepersonal in anderen Bereichen tätig werden musste. In dieser Phase war das Gesundheitssystem nur deshalb stabil, weil sehr rasch andere elektive Gesundheitsleistungen heruntergefahren wurden. Ob dies aus der Metaperspektive tatsächlich von Nutzen war, lässt sich nur sehr schwer beurteilen, denn ex post wurde klar, dass viele Menschen aus Angst vor einer SARS-CoV2-Infektion nicht ins Spital gegangen sind, obwohl es aufgrund ihres Gesundheitszustandes indiziert gewesen wäre. Offensichtlich haben manche dadurch Gesundheitsschäden davongetragen.

Da man nach jedem Lockdown anstreben musste, diese postponierten elektiven Patienten zeitnah zu operieren, gab es für das Spitalspersonal keine Erholungsphase nach COVID-19, sondern es ging von einem Vollbetrieb in den nächsten. Die Herausforderungen und Belastungen waren und sind sowohl für die Ärzte als auch für das Pflegepersonal wirklich enorm.

Kröll: Wie ausgelastet waren die für die Behandlung von infizierten Patienten zur Verfügung gestellten Intensivbehandlungsbetten? Wie viele Betten des LKHUniv.-Klinikum Graz wurden eigentlich für infizierte Patienten zur Verfügung gestellt? Wie viele Betten hättest Du noch für die Behandlung von COVID-19Patienten bereitstellen können? Und wäre dafür auch ausreichend und vor allem auch adäquat ausgebildetes Pflegepersonal zur Verfügung gestanden? Wurde in Deinem Verantwortungsbereich eigentlich jemals an Triage gedacht, so wie das vielfach in den Medien kolportiert worden war?

Toller: Bezüglich der Auslastung jener Intensivbehandlungsbetten, die für COVID-19-Patienten zur Verfügung gestellt worden sind, hat sich auch gezeigt, wie weit Theorie und Praxis auseinanderklaffen. Auf der einen Seite war zu Beginn der Pandemie nicht bekannt, wie viele Intensivbetten man tatsächlich benötigen werde; es waren zwar Bilder aus Italien bekannt und dies führte am Beginn der Pandemie dazu, dass eine sehr große Zahl an Intensivbehandlungsbetten freigehalten wurde. Bald hat es sich aber herausgestellt, dass eine derart große Zahl an Intensivbehandlungsbetten nicht benötigt wird. Freie Intensivbehandlungsbetten waren natürlich nur deshalb in großer Zahl verfügbar, weil zahlreiche elektive Patienten, die postoperativ auch ein Intensivbehandlungsbett benötigt hätten, nicht operiert wurden.

Die Zahl der für die Behandlung von COVID-19-Patienten am LKHUniv.-Klinikum Graz zur Verfügung gestellten Intensivbehandlungsbetten 
hat sich in der weiteren Phase der Corona-Pandemie durch strategische Maßnahmen geändert. Es wurde errechnet, wie viele Intensivbehandlungsbetten in der Steiermark theoretisch benötigt werden könnten. Die verfolgte Strategie war dabei in der Phase der ersten Corona-Welle anders als in der Phase der zweiten Corona-Welle: In der Phase der ersten Corona-Welle wurden einzelne Krankenanstalten, so auch das LKH.-Univ.-Klinikum Graz als „Corona-Krankenanstalten“ klassifiziert und somit alle krankenhauspflichtigen COVID-19-Patienten aus der gesamten Steiermark auch in eines dieser Krankenhäuser transferiert. Zu diesem Zeitpunkt entstand auch noch die Situation, dass andere Krankenhäuser, die eigentlich nicht für die Behandlung von COVID-19-Patienten vorgesehen waren, aus Angst, es könnten noch viel mehr Menschen infiziert werden, ihre Intensivbehandlungsbetten ebenfalls freihielten. So entstand eine Situation, dass es auf der einen Seite Krankenanstalten gab, die überhaupt keine Belegung mit COVID-19-Patienten aufwiesen, und in anderen Krankenanstalten eine extrem schwierige Situation vorlag, weil eine hohe Auslastung und damit ein hoher Belag von Intensivbehandlungsbetten gegeben war.

Diese Strategie wurde in der Phase der zweiten Corona-Welle dahingehend umgestellt, dass nun alle Krankenhäuser in der Steiermark verpflichtet wurden COVID-19-Patienten zu behandeln, auch die konfessionellen Krankenanstalten. Das hätte dann zwar grundsätzlich bedeuten können, dass die Belastung für das LKH-Univ.-Klinikum Graz geringer hätte werden können, allerdings war die zweite Welle dadurch charakterisiert, dass die Zahl an infizierten Menschen und somit auch Intensivpatienten viel höher war als während der ersten Phase. Zeitweise waren in der Steiermark mehr als 120 der theoretisch 150 für COVID-19-Patienten vorhandenen Intensivbetten belegt (von insgesamt 2500 Intensivbehandlungsbetten in den Krankenanstalten Österreichs). Dadurch verlief auch die zweite Welle der Infektion für unser Klinikum trotz der vorher beschriebenen veränderten Strategie sehr angespannt.

Insgesamt muss man festhalten, dass im Rahmen der bislang abgelaufenen Corona-Pandemie Flexibilität gefragt ist. Wenn viele Menschen infiziert und die Krankenanstalten überfüllt sind, dann hat sich ein Vorgehen wie mit der geänderten Strategie sehr bewährt, nämlich dass möglichst viele Krankenhäuser an der Versorgung der COVID-19-Patienten beteiligt sind. Dadurch war es auch möglich, dass der Umgang mit den Schutzkleidungen sowie den Behandlungsstrategien dieser Patienten beinahe schon zur Routine geworden ist und dadurch auch etwas leichter bewältigt werden konnte. Hätte man diese Strategie in der ersten Phase eingesetzt, wäre das aus meiner Sicht mit erheblichen Nachteilen für die Patienten verbunden gewesen, weil es kaum möglich gewesen wäre, eine gute, dem Stand 
der medizinischen Wissenschaften adäquate Versorgung in allen beteiligten Krankenanstalten zu gewährleisten, eben weil es auch nicht möglich gewesen wäre, ausreichend Routine im Umgang mit dieser Erkrankung zu gewinnen. Welche Strategien man in Zukunft wählen wird, muss sich aus der jeweiligen Situation heraus weisen.

Auch im Zusammenhang mit dem Personal ist es anfangs extrem wichtig, die Behandlung dieser Patienten auf wenige Bereiche zu konzentrieren, um damit auch eine hohe Expertise zu erhalten. Wenn dann in einer späteren Phase der Corona-Pandemie das Wissen um die Behandlung schon sehr groß ist und auch eine gewisse Routine im Umgang damit gewonnen werden konnte und auch die Kommunikation zwischen den einzelnen Stationen, auf denen COVID-19-Patienten behandelt werden, adäquat gegeben ist, dann wird sich sicherlich die zweite Strategie als sehr geeignet erweisen.

Das LKH-Univ.-Klinikum Graz war glücklicherweise nie in der Situation, Patienten hinsichtlich einer Verlegung auf eine Intensivstation triagieren zu müssen; dennoch wurde viel Zeit in strategische Überlegungen investiert, falls man tatsächlich an die Grenzen der Versorgungsmöglichkeit kommen sollte. Innerhalb der Ärzteschaft und auch in unserem Behandlungsteam gab und gibt es heterogene Meinungen zum Umgang mit dieser Thematik.

Kröll: Zumindest in der ersten Phase der Pandemie wurden elektive chirurgische Eingriffe postponiert. Deine Abteilung betreut mit herz-, lungen- und gefäßkranken Menschen eine sehr spezielle Patientengruppe, bei denen einerseits die Operationsindikation ziemlich dringlich ist und bei denen andererseits bei Nichtintervention die Wabrscheinlichkeit zu versterben sehr hoch ist. Wie war das auf Deiner Abteilung? Wie hast Du das als Leiter dieser Abteilung erlebt? Ist bekannt wie viele Patienten auf den genannten chirurgischen Abteilungen verstorben sind, weil sie nicht chirurgisch und/oder interventionell versorgt werden konnten?

Toller: Die Sorge vor der durch Corona nicht möglichen routinemäßigen Versorgung von Patienten mit Herzerkrankungen während der Pandemie stellte sich als nicht so gravierend heraus, wie ursprünglich gedacht; dies scheint damit zu tun zu haben, dass insgesamt weniger Patienten mit Erkrankungen des Herz-Kreislaufsystems in das Krankenhaus gekommen sind, offensichtlich aus Angst vor einer Corona-Infektion. Anderseits weiß man leider auch, dass einige dieser Patienten, bei denen eine Intervention oder Operation notwendig gewesen wäre, aus Angst vor einer Ansteckung im Spital zu Hause verstorben sind. Dieser Effekt konnte auch durch 
eine Untersuchung der Medizinischen Universität Graz bestätigt werden. Retrospektiv wurden aber alle Patienten, die wegen einer dringlichen operativen Intervention am Herzen stationär aufgenommen worden waren, auch tatsächlich versorgt.

Auch die Zahl an Patienten, die wegen anderer chirurgischer Eingriffe ins Krankenhaus kamen, war in der Pandemiezeit nicht besonders hoch. Insbesondere wurde versucht, elektive Operationen zu vermeiden, von denen man weiß, dass die Patienten mit hoher Wahrscheinlichkeit postoperativ ein Intensivbett brauchen werden. Wurden solche Patienten dennoch operiert, blieben sie so kurz als möglich auf der Intensivstation oder wurden überhaupt in einem Aufwachraum behandelt und möglichst bald wieder auf die Normalstation verlegt.

Kröll: Die Corona-Pandemie hat insbesondere bei Jugendlichen, aber auch generell zu einem überdimensionalen Anstieg von psychischen Krankheitsbildern, insbesondere von Depressionen geführt. Konntest Du davon auch bei Deinen Mitarbeitern etwas bemerken? Gab es Burn-outs unter den Mitarbeitern oder zumindest eine fehlende Motivation aufgrund der Belastung bei der Betreuung und Behandlung dieser speziellen Patientengruppe?

Toller: Man hat gesehen, dass sich während des Lockdowns das Leben zwischen Wohnung und Arbeitsstätte abspielt und dass das speziell für Mitarbeiter, die alleine leben problematisch war. Nach einer initialen Phase des maximalen Engagements in der Behandlung und Betreuung von COVID-19-Patienten kam es im weiteren Verlauf der Corona-Pandemie dann zu Verstimmungen, Müdigkeit, Burn-out und auch zum Wunsch des Arbeitsplatzwechsels. Ich bin daher überzeugt, dass ein Burn-out in solchen Bereichen, wo man stundenlang oft ohne Pausen in die Behandlung und Betreuung dieser Patienten involviert ist, durchaus ernst zu nehmen ist. Ex post kann ich nur dafür plädieren, dass, sollte sich eine solche Pandemie erneut ereignen, man die Mitarbeiter möglichst frühzeitig auch psychologisch unterstützt.

$\mathrm{Ob}$ die Lockdowns tatsächlich erforderlich waren, kann ich nicht beurteilen; aber aus meiner Perspektive ist festzuhalten, dass wir alle im Krankenhaus - ärztliche und nicht-ärztliche Gesundheitsberufe - gelernt haben mit dieser Erkrankung umzugehen, ohne dass es zu einer signifikanten Steigerung an infizierten Mitarbeitern gekommen wäre. Ich bin überzeugt, dass es mit entsprechenden Rahmenbedingungen und Schulungen möglich sein müsste, eine solche Krise (Mai 2021) auch ohne Lockdowns zu bewältigen, ohne gleich das gesamte öffentliche Leben (Wirtschaft, Universität, Schulen, Kulturbetriebe etc.) herunterzufahren. 
Kröll: Die Corona-Pandemie hat nicht nur in Österreich zu einer Spaltung der Gesellschaft in Befürworter und Gegner der von den Behörden angeordneten Maßnahmen geführt. Wie siehst Du nun ex post diese Maßnahmen aus der Sicht eines Klinikvorstandes, aber auch aus der Sicht eines klinisch tätigen Arztes? Waren all diese Maßnahmen gerechtfertigt oder hätte man etwas anderes machen sollen und/oder können? Waren die verordneten Lockdowns gerechtfertigt? Machen Testungen einen Sinn für den Besuch diverser Dienstleistungen? Oder anders gefragt: Welche Maßnahmen machen aus Deiner Perspektive Sinn?

Toller: Eine so wie derzeit praktizierte generelle Verpflichtung zur regelmäßigen Testung aller Spitalsmitarbeiter halte ich persönlich für nicht (mehr) sinnvoll, weil im LKH-Univ.-Klinikum Graz sehr viele Mitarbeiter geimpft sind. Manche Tests sind positiv, ohne dass der Mitarbeiter irgendwelche Symptome hat und nichts über dessen mögliche Infektiosität bekannt ist. Ich bin deshalb überzeugt, dass sich diese Antigentests aufhören werden, denn meiner Meinung nach machen die Impfung und die erforderliche Auffrischungsimpfung die Gesamtsituation wesentlich sicherer als regelmäßige Testungen der einzelnen Mitarbeiter; testen sollte man vor allem jene, die nicht geimpft sind; ferner glaube ich, dass die Einhaltung und Durchführung entsprechender hygienischer Maßnahmen von zentraler Bedeutung ist.

Kröll: Die Verfügbarkeit von Impfstoffen scheint die Situation etwas zu entspannen. Zumindest können damit nicht nur Risikogruppen, sondern auch, soweit Impfstoff vorhanden, auch Impfwillige immunisiert werden. Heftig in diesem Zusammenhang diskutiert wird die Frage des Impfzwanges. Wie siehst Du das Thema Impfzwang im Zusammenhang mit dem Gesundheitspersonal? Sollte dieses verpflichtet geimpft werden? Oder ist aus Deiner Perspektive das Risiko Nebenwirkungen zu erleiden, wie die bereits bekannte Neigung zu Sinusvenenthrombosen, zu hoch, um eine generelle Impfpflicht bei Angehörigen der diversen Gesundheitsberufe zu fordern?

Toller: Einen generellen Impfzwang für das Gesundheitspersonal halte ich für kritisch, obwohl es diesen in manchen Spitälern bei Personal-Neueinstellungen bezüglich anderer Viruserkrankungen (z. B. Masern) bereits gibt. Mit Zwang wird man Impf-Gegner nicht erreichen. Ich bin aber andererseits überzeugt, dass es in gewissen Bereichen z. B. bei Fluglinien oder auch in den diversen Urlaubsdomizilen ohnehin so werden wird, dass eine Impfung zur (Ein)reise nachgewiesen werden wird müssen. 
Bezüglich des Gesundheitspersonals erscheint es interessant, dass gerade in jenen Bereichen, in denen COVID-19-Patienten behandelt worden sind, die Impfskepsis sehr groß ist. Gerade diese Mitarbeiter, die eigentlich dem größten Risiko ausgesetzt waren, waren bezüglich einer Impfung zurückhaltend. Möglicherweise auch deshalb, weil sich gezeigt hat, dass adäquate Schutzmaßnahmen auch schon vor Vorhandensein einer Impfung die Übertragung einer Infektion verhindern konnten. Aus meiner Sicht wäre es daher sinnvoller, adäquate hygienische Rahmenbedingungen einzufordern, und wie auch gezeigt werden kann, sind diese für eine Prävention sehr sinnvoll und effektiv. Man müsste dann nicht von einem Impfzwang ausgehen, der eigentlich nur dazu führt, dass sich Menschen gerade wegen des verordneten Zwanges eben nicht impfen lassen.

Bezüglich der Nebenwirkungen, insbesondere die Thromboseneigung betreffend, wurde die Öffentlichkeit im Rahmen von COVID-19 durch die öffentlichen Medien quasi zum ersten Mal mit Prozessen von Medikamentenentwicklung, experimentellen und klinischen Studien und auch potenziellen Nebenwirkungen „live“ konfrontiert, das Ganze noch ergänzt durch die gigantischen Kommunikationsmöglichkeiten der heutigen Zeit. Während die Einschätzung und Beurteilung neuer Medikamente für Mediziner zum Alltag gehört, ist ein großer Teil der Bevölkerung damit schlichtweg überfordert. In der Medizin muss bei jeder Verordnung eines Medikamentes eine Nutzen-Risiko-Analyse vorgenommen werden und es gibt kein Medikament ohne Nebenwirkungen. Wenn man sich das Risikopotenzial einer Corona-Impfung betrachtet und erkennt, dass bei zwei Millionen durchgeführten Impfungen 140 Thrombosen auftraten, klingt das vielleicht viel, ist es aber nicht und das Risiko des Einzelnen eine Thrombose $\mathrm{zu}$ erleiden ist verschwindend gering aber eben nicht null. Gleichzeitig ist man durch die Impfung geschützt und kann keine (viel häufigere!) Thrombose durch eine eventuelle COVID-19-Erkankung erleiden. Das Nutzen-Risiko-Verhältnis der Impfung ist also o. K. Vergleicht man damit das Thromboserisiko durch die Einnahme einer Anti-Baby-Pille, so ist diese 1000-fach höher. Ich kann mir nicht vorstellen, dass es viele Frauen gibt, die deshalb auf die Einnahme der Pille verzichten würden. Diese Überlegungen treffen für alle Medikamente zu. Es gibt kein Medikament, das keine Nebenwirkungen hat, sondern es ist immer nur eine Frage der Häufigkeit. Selbstverständlich ist eine eingetretene Nebenwirkung für den einzelnen Menschen, den es trifft, ein katastrophales Ereignis, obwohl sich mit Blick auf die Sinusvenenthrombose auch gezeigt hat, dass, wenn sie rechtzeitig erkannt wird, gut behandelt werden kann. Aus meiner Perspektive ist das Risiko an COVID-19 zu erkranken, das Risiko einen schweren Verlauf zu erleiden und schließlich das Risiko an den Folgeschä- 
den (Post-COVID-Syndrom) zu leiden wesentlich höher als das Risiko, dass durch die Corona-Impfung schwere Nebenwirkungen auftreten. Aus meiner Sicht ist daher die Nebenwirkung kein Grund sich nicht impfen zu lassen.

Kröll: Ich danke Dir für Deine Bereitschaft zu diesem Interview und wünsche Dir weiterhin alles Gute. 
